\title{
A Three-Dimensional Inverse Finite-Element Method Applied to Experimental Eddy-Current Imaging Data
}

\author{
Manuchehr Soleimani ${ }^{1}$, William R. B. Lionheart ${ }^{1}$, Antony J. Peyton ${ }^{1}$, Xiandong Ma ${ }^{1}$, and Stuart R. Higson ${ }^{2}$ \\ ${ }^{1}$ University of Manchester, Manchester M60 1QD, U.K. \\ ${ }^{2}$ Corus UK Ltd. RD\&T, Teesside Technology Centre, Grangetown, Middlesbrough TS6 6US, U.K.
}

\begin{abstract}
Eddy-current techniques can be used to create electrical conductivity mapping of an object. The eddy-current imaging system in this paper is a magnetic induction tomography (MIT) system. MIT images the electrical conductivity of the target based on impedance measurements from pairs of excitation and detection coils. The inverse problem here is ill-posed and nonlinear. Current state-of-the-art image reconstruction methods in MIT are generally based on linear algorithms. In this paper, a regularized Gauss-Newton scheme has been implemented based on an edge finite-element forward solver and an efficient formula for the Jacobian matrix. Applications of Tikhonov and total variation regularization have been studied. Results are presented from experimental data collected from a newly developed MIT system. The paper also presents further progress in using an MIT system for molten metal flow visualization in continuous casting by applying the proposed algorithm in a real experiment in a continuous casting pilot plant of Corus RD\&T, Teesside Technology Centre.
\end{abstract}

Index Terms-Edge finite-element method, inverse problems, magnetic induction tomography, total variation regularization.

\section{INTRODUCTION}

$\mathbf{M}$ AGNETIC INDUCTION TOMOGRAPHY (MIT) is a new modality for medical, industrial, and geophysical imaging [1]-[3]. The problem of MIT image reconstruction is similar to the inverse eddy-current problem of nondestructive testing (NDT) [4]-[7]. The measurement data are the mutual inductances between pairs of coils. The contact-less nature of this type of tomography makes the technique of interest for noninvasive and nonintrusive applications. The technique operates as follows. Passing an alternating current through the excitation coil(s) produces a primary magnetic field. When this magnetic field interacts with either a conductive and/or a magnetic object, a secondary magnetic field is created. The sensing coils can then detect this secondary field. As the secondary field depends on the materials present, the measured induced voltage is a nonlinear function of their electrical properties, e.g., conductivity and permeability. In comparison with conventional eddy-current methods, MIT is significant in that it measures the magnetic coupling, rather than self-inductance, using sensors that are distributed around the periphery, and therefore is able to image the distribution of materials inside a region of interest. Image reconstruction is an inverse problem where the measured voltages are given and the spatial distribution of electromagnetic properties of the object material need to be found. Linear reconstruction methods [8], [9] are fast and can be successfully applied to isolated objects. In general, it is necessary to use nonlinear reconstruction methods. The most commonly used method for the image reconstruction in electrical impedance tomography (EIT) is a regularized Gauss-Newton method. This method has been studied for magnetostatic permeability tomography using simulated data in [10]. Each step of such an iterative method is a linear problem. This paper presents such a method to the inverse problem of MIT using experimental test data. To be practically usable, it is very important for an image reconstruction method

Digital Object Identifier 10.1109/TMAG.2006.871255 to be tested with experimental data. In this paper, we present the reconstruction results for laboratory tests as well as experimental results from a practical test in molten steel flow visualization [8]. Reconstruction of the MIT images requires a forward solver so that predicted data can be compared with measured data. The forward problem in MIT is the eddy-current approximation of Maxwell's equations, which involves computation of the vector fields. The edge finite-element method (FEM) has advantages over nodal FEM for vector field computation in eddy-current problems [11], and it is a powerful tool for simulation of the forward problem in MIT [12], [13]. We have implemented a flexible edge-based finite-element eddy-current solver for the forward problem in MIT using a magnetic vector potential formulation [13]. An efficient formulation for the Jacobian matrix is used [14]-[16]. The method requires only two solutions of the forward problem for each coil pair, first exciting one coil and then the second.

In order to stabilize the ill-posed inverse problem, regularization techniques are required. A common choice is Tikhonov regularization that assumes a smooth conductivity distribution [10]. The Tikhonov regularized Gauss-Newton method is not ideally suited to problems with a sharp transition in conductivity between phases. The total variation (TV) functional assumes an important role in the regularization of inverse problem arising in many disciplines [17], after its initial introduction in image restoration. The use of such a functional as a regularization penalty term allows the reconstruction of discontinuous profiles. This is a desirable property where there are sharp edges with large transitions of the conductivity profile. TV has been applied to the general form of the elliptic inverse problem [18], and EIT [19]. In this paper, we further examine the advantage of TV regularization in separation of high contrast conductivity objects close to each other with experimental test examples.

This paper is organized as follows. In Section II, we introduce a newly developed MIT system used for the experimental tests. In Section III, we describe our edge FEM formulation of the forward problem. A sensitivity analysis using an efficient formulation will be given in Section IV, where we also present an 


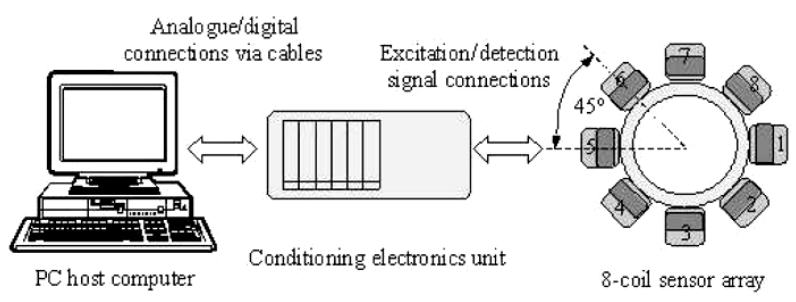

Fig. 1. Diagram of the MIT system used in this study.

example a three-dimensional (3-D) sensitivity map. In Section V, we explain the nonlinear inverse solver and with commonly used Tikhonov and with TV regularization. In Section VI, the experimental results will be presented using these new methods. The experimental reconstruction presented in Section VI-A are among the first experimental MIT reconstructions using an FE-based inversion technique. In Section VI-B, we also present the molten steel flow images from an MIT experimental system.

\section{MIT SYSTEM DESCRIPTION}

The MIT technique has been considered in situations where the target materials of interest are characterized by electrical conductivity, as is the case of monitoring of the steel flow in a continuous casting nozzle [8].

Fig. 1 shows a diagram of the main parts of the MIT system. The hardware consists of a sensor array, waveform generation; high current buffer for excitation; programmable gain amplification and in-phase/quadrature demodulation for detection and analog to digital conversion. The waveform generator outputs a $5 \mathrm{kHz}$ sinusoidal current. The electronic hardware also allows the switching of the alternating current signal to each coil for excitation sequentially, controls the gain selection for the induced voltage amplification, and selects the demodulated signal components for analog-to-digital conversion. Full details of the electronic hardware are beyond the scope of this paper, but have been reported elsewhere [20].

The sensor array contains eight wire wound coils (50 turns, $50 \mathrm{~mm}$ diameter). The distance between centers of two opposite coils is $160 \mathrm{~mm}$. All the coils are equally spaced at intervals around the periphery of the object to be imaged. The region of interest for the imaging is a cylinder with radius $0.07 \mathrm{~m}$, length $0.10 \mathrm{~m}$ centered at $(0,0,0)$, and a relative permeability of 1 (labeled C1). Each coil is excited in turn and the induced voltages are measured in the remaining coils. The real part of the induced voltage (in-phase with the excitation) has been used for the conductivity reconstruction.

\section{FORWARD PROBLEM}

The forward problem is a general eddy-current problem and is solved using an edge FEM method in terms of magnetic vector potential (A) [11], [13]. The forward model has been validated in a previous study [13]. Given $\mathbf{E}=-\mathrm{i} \omega \mathbf{A}$ and $\mathbf{B}=\nabla \times \mathbf{A}$, we have

$$
\nabla \times(\nu \nabla \times \mathbf{A})=\mathbf{J}_{\mathbf{s}}
$$

in nonconductive regions, where $\nu=1 / \mu$. In the conductive region we have

$$
\nabla \times(\nu \nabla \times \mathbf{A})+\mathrm{i} \omega \sigma \mathbf{A}=\mathbf{J}_{\mathbf{s}} .
$$

Here $\sigma$ is electrical conductivity and $\omega$ is angular frequency. Let us denote by $L_{i}$ the nodal scalar basis function. In edge FEM on a tetrahedral mesh, a vector field is represented using a basis of vector valued functions $\mathbf{N}_{i j}$ associated with the edge between nodes $i$ and $j$

$$
\mathbf{N}_{i j}=L_{i} \nabla L_{j}-L_{j} \nabla L_{i} .
$$

Using edge finite elements

$$
\begin{aligned}
\int_{\Omega}(\nabla \times \mathbf{N} \nu \cdot \nabla \times \mathbf{A}) d V+\int_{\Omega_{e}}(\mathrm{i} \omega \sigma \mathbf{N} \cdot \mathbf{A}) d V \\
=\int_{\Omega_{c}}\left(\mathbf{N} \cdot \mathbf{J}_{\mathbf{s}}\right) d V
\end{aligned}
$$

where $\mathbf{N}$ is any linear combination of edge basis functions, $\Omega$ is the entire region, $\Omega_{e}$ is the eddy-current region, and $\Omega_{c}$ is the current source region. Here electric vector potential $\mathbf{T}_{s}$ has been used to simulate the current source $\nabla \times \mathbf{T}_{s}=\mathbf{J}_{s}$ as described [11]. The incomplete Cholesky conjugate gradient (ICCG) method has been used to solve the linear system of equations arising from the forward problem [11]. In each iteration of the inverse problem, we solve the forward problem and the starting point for ICCG iteration is the latest value of magnetic vector potential $\mathbf{A}$ in previous step.

\section{SENSITIVITy ANALYSIS}

The sensitivity formula has been derived earlier from the general form of Maxwell's equations and for the special case of inverse eddy-current problem of MIT [14], [16]. A sensitivity formula that reflects the high conductivity changes has been used for eddy-current NDT (see for example [4]).

The general form of the sensitivity formula when the coil 1 is an excitation coil and coil 2 is a sensing coil, by ignoring second-order terms is [14]

$$
\begin{aligned}
\int_{\Gamma} \delta \mathbf{E}_{1} & \times \mathbf{H}_{2} \cdot \mathbf{n} d S \\
& =\int_{\Omega}-\mathrm{i} \omega \delta \mu \mathbf{H}_{1} \cdot \mathbf{H}_{2}+(\delta \sigma+\mathrm{i} \omega \delta \varepsilon) \mathbf{E}_{1} \cdot \mathbf{E}_{2} d V
\end{aligned}
$$

where the left-hand side is representing sensing and excitation by surface integral on surface $\Gamma$ and the right-hand side is the volume integral over the perturbed region $\Omega . \mathbf{H}_{1}$ and $\mathbf{E}_{1}$ are the magnetic and electric fields when coil 1 is excited and $\mathbf{H}_{2}$ and $\mathbf{E}_{2}$ are the magnetic and electric fields when coil 2 is excited. Similar sensitivity formulas have been discovered in the literature of numerous electromagnetic sensing methods and justified with varying degrees of mathematical rigor. In the NDT context, [21] is an early example. With the $\mathbf{A}, \mathbf{A}$ formulation and using edge FEM, the sensitivity to a change in the conductivity of the conducting region can be calculated using the results of the forward solver, given no change in permeability and permittivity values in (5). Using the edge element basis functions $\mathbf{N}_{e}$, the potential $\mathbf{A}$ inside each element can be expressed as follows:

$$
\mathbf{A}=\mathbf{N}_{e} \mathbf{A}_{e}
$$




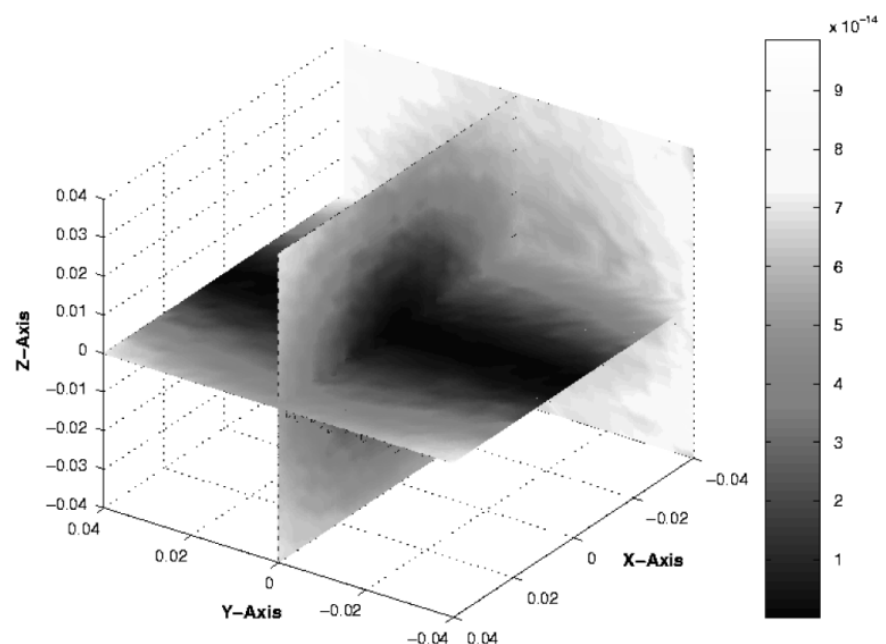

Fig. 2. Sensitivity plot for two opposite coils in 3-D for unit current density in excitation coil, the sensitivity values for the real part of the induced voltage are in $\mathrm{VmS}^{-1}$, dimensions are in $\mathrm{m}$.

where $\mathbf{A}_{e}$ are defined along edges. With that, the sensitivity term for each element as follows:

$$
\frac{\partial V_{i j}}{\partial \sigma_{k}}=-\frac{\omega^{2}}{I_{i} I_{j}} \mathbf{A}_{e}^{i}\left(\int_{\Omega_{e k}} \mathbf{N}_{e} \cdot \mathbf{N}_{e}^{T} d V\right)\left(\mathbf{A}_{e}^{j}\right)^{T} .
$$

Equation (7) gives us sensitivity of the induced voltage pairs $V_{i j}$ of coils of $i, j$ with respect to an element and $\Omega_{e k}$ is the volume of element number $k$ and $I_{i}$ and $I_{j}$ are excitation currents for the coils. For small conductivity perturbations, the formula presented in [4] reduces to (7). A similar formula has also been presented by [6] for the NDT problem.

In the edge FEM software we developed for image reconstruction, we calculate $\mathbf{A}$ in all elements by (6). We can then use (7) simply for region $\Omega_{f}$, which includes more than one finite element. Then the computation of the Jacobian matrix is a matrix vector multiplication for each measurement. The sensitivity map changes with the background conductivity [22]. With a conductive background close to the surface, we have higher eddy currents, and consequentially those areas have higher sensitivity. Sensitivity also depends on the geometrical configuration of the sensing and exciting coils. For example, using a single frequency and fixed shape of the conductive background, for high conductivity the higher eddy-current density region is very small and changes in regions very close to the boundary are more easily detectable. When the conductivity decreases, the area of high sensitivity spreads toward the center. Finally, when the conductivity goes to zero, the more sensitive area is no longer affected by the conductive background shape and it is only affected by the geometrical configuration of the sensing and exciting coils. Fig. 2 shows a 3-D sensitivity map for two opposite coils centered at $(0,-0.08)$ and $(0,0.08)$, the background is $\mathrm{C} 1$ with conductivity $4 \mathrm{Sm}^{-1}$ and frequency is $1 \mathrm{MHz}$. As it can be seen in this figure, the sensitivity reduces toward the central area as the eddy current is higher near the boundary of the conductive object.

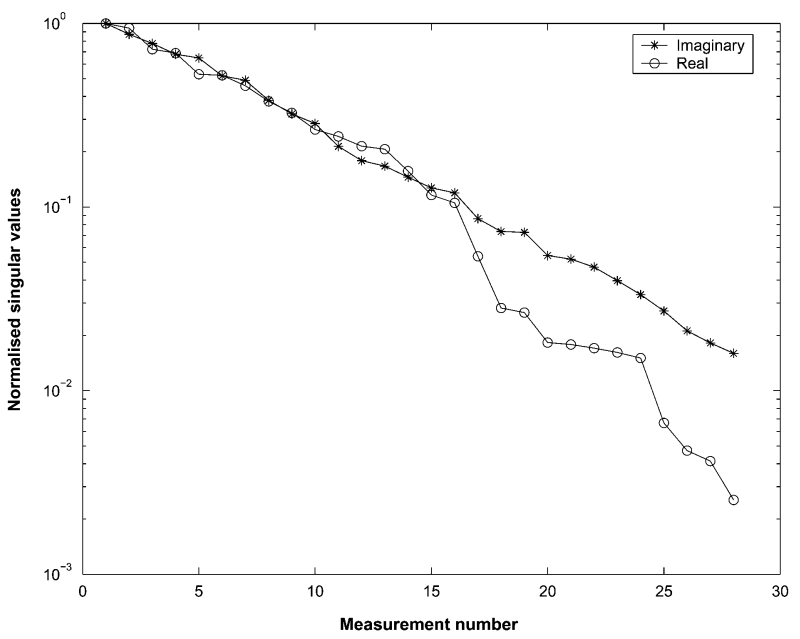

Fig. 3. Singular values of the Jacobian matrix ( $\mathbf{J})$ for the real and imaginary part of the measurement voltages on a logarithmic scale (background is $\mathrm{C} 1$ with conductivity $4 \mathrm{Sm}^{-1}$ and frequency $1 \mathrm{MHz}$ ).

\section{A. Jacobian Matrix}

In the Jacobian matrix, each row is the calculated sensitivity term for an element for all measurements. Fig. 3 shows the distribution of the singular values of the real part of the Jacobian matrix. In a noise- and error-free situation, it is possible to reconstruct an image with up to 28 parameters. In real data and with measurement errors, according to the discrete Picard criterion, some of the small singular values may not reliably contribute to the image reconstruction. It is worth noticing that some of these small singular values may represent important and desirable parts of the images which may not be reconstructed due to noise and error in the measurement.

To illustrate the degree of ill conditioning in the problem, the singular values are plotted on the log scale (Fig. 3). The roughly linear decay of the first 28 singular values shows that the problem is ill-posed. Here the background conductivity is the cylinder $\mathrm{C} 1$ with homogeneous conductivity of $4 \mathrm{Sm}^{-1}$ and frequency is $1 \mathrm{MHz}$. In medical MIT, the in-phase component of the received signal are used to reconstruct conductivity of the tissue [23]. The quality of imaging depends on the precision with which the real part can be measured. In Fig. 3, one can see that using the imaginary part of receiving signal (with this particular conductivity and frequency) the inverse problem is actually better posed than using the real part. In practical terms, measuring the imaginary part (detecting a very small signal due to the conductive object, in-phase with a very large primary signal) is a very difficult task for medical MIT applications.

Fig. 4 shows the singular values of the Jacobian for background $\mathrm{C} 1$ with conductivity $5.8 \times 10^{7} \mathrm{Sm}^{-1}$ and frequency $5 \mathrm{kHz}$. This figure shows that using real part or imaginary part to reconstruct conductivity is more ill-posed that the problem of Fig. 3, but the ill-posedness of the problem using real data and imaginary data is similar.

\section{INVERSE PROBLEM}

A regularized Gauss-Newton method is used to solve the inverse problem. We denote by $V_{m}$ the measurement data, and by $F$ the forward operator, which takes the vector of degree of 


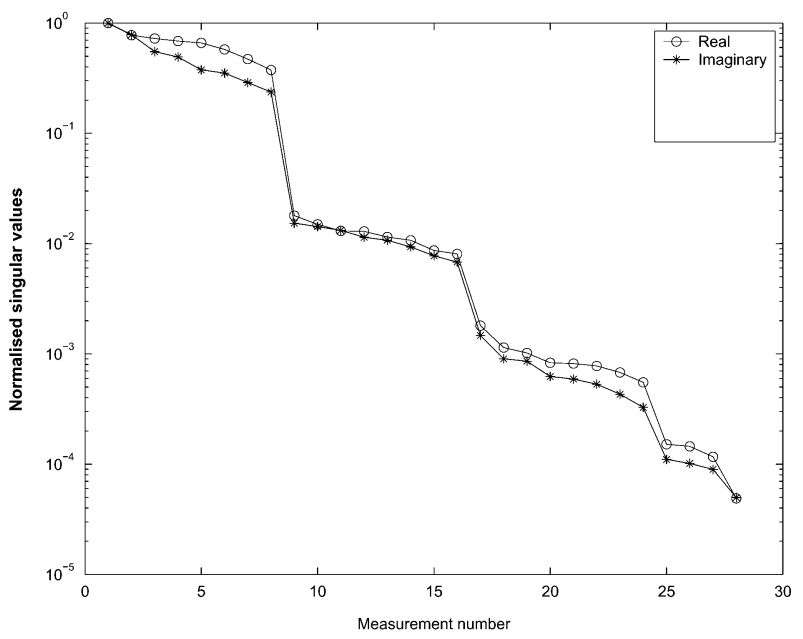

Fig. 4. Singular values of the Jacobian matrix (J) for the real and imaginary part of the measurement voltages on a logarithmic base (background is $\mathrm{C} 1$ with conductivity $5.8 \times 10^{7} \mathrm{Sm}^{-1}$ and frequency $5 \mathrm{kHz}$ ).

freedom in the conductivity $\sigma$ and calculates the corresponding voltages. The inverse solver is an optimization algorithm that finds a $\boldsymbol{\sigma}$ to minimize a suitable error functional. Reformulation of the inverse problem to include prior information is known as regularization and it can be expressed as a minimization of the functional

$$
f(\boldsymbol{\sigma})=\left\|V_{m}-F(\boldsymbol{\sigma})\right\|^{2}+\alpha^{2} G(\boldsymbol{\sigma})
$$

where $\alpha^{2} G(\boldsymbol{\sigma})$ is a penalty term. The Jacobian matrix $\mathbf{J}$ is a discretization of $F^{\prime}(\boldsymbol{\sigma})$. The regularization parameter $\alpha$ controls the tradeoff between fitting the data and reducing the penalty term. For a well-chosen $G, f$ will have a critical point which is the minimum, at this minimum $\nabla f=0$. A typical example of a discrete form of a penalty term is $G(\boldsymbol{\sigma})=\|\mathbf{R} \boldsymbol{\sigma}\|^{2}$ for a matrix $\mathbf{R}$ approximating a differential operator. A more detailed study of the iterative Gauss-Newton step to solve nonlinear ill-posed problem can be seen in [24]. As an iterative reconstruction algorithm, we start our regularized Gauss-Newton method with an initial conductivity distribution $\boldsymbol{\sigma}_{0}$. The forward problem is solved and the predicted voltages are compared with the calculated voltages from the forward model. The conductivity is then updated. The process is repeated until the predicted voltages from the finite-element method agree with the calculated voltages from the finite-element model to measurement precision. In the nonlinear steps, the Jacobian matrix is also updated in each step. Solving this minimization problem by Gauss-Newton leads to linear steps, each of which is the regularized step of the linearized problem (for the simple form of $G$ above)

$$
\begin{array}{r}
\delta \boldsymbol{\sigma}_{n+1}=\left(\mathbf{J}_{n}^{T} \mathbf{J}_{n}+\alpha^{2} \mathbf{R}^{T} \mathbf{R}\right)^{-1}\left(\mathbf{J}_{n}^{T}\left(\mathbf{V}_{m}-\mathbf{F}\left(\boldsymbol{\sigma}_{n}\right)\right)\right. \\
\left.-\alpha^{2} \mathbf{R}^{T} \mathbf{R} \boldsymbol{\sigma}_{n}\right)
\end{array}
$$

where $n$ is the iteration step. For $n=1$, this is a linear reconstruction algorithm.

\section{A. Tikhonov Regularization}

A natural assumption is that the conductivity is fairly smooth, which can be expressed by demanding that $G(\boldsymbol{\sigma})=\|\mathbf{R} \delta \sigma\|^{2}$

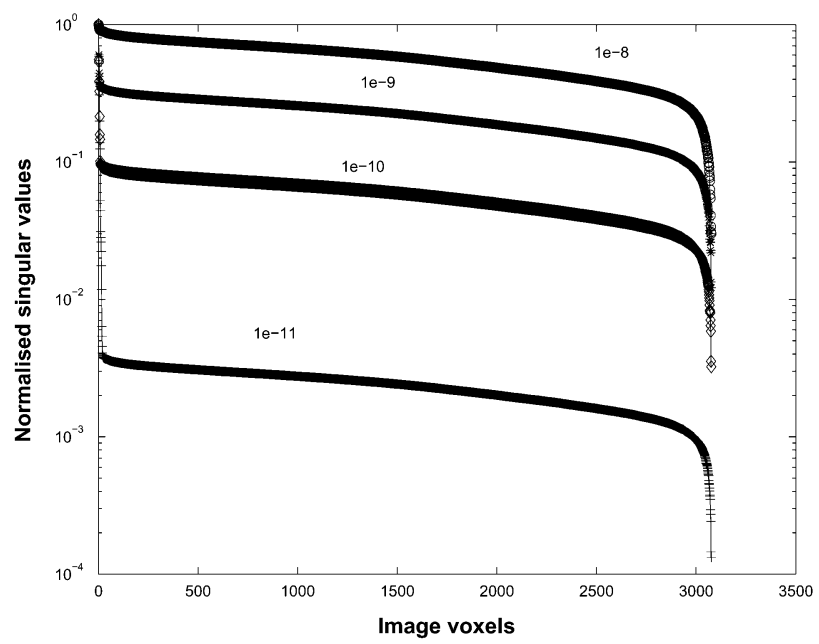

Fig. 5. Singular value distribution for $\mathbf{J}^{T} \mathbf{J}+\alpha^{2} \mathbf{R}^{T} \mathbf{R}$ for cylinder $\mathrm{C} 1$ for different regularization parameters (different values for $\alpha^{2}$ ).

is not too large, where the regularization matrix $\mathbf{R}$ is a difference operator between neighboring pixels. This results in (generalized) Tikhonov regularization, which penalizes extreme variations in conductivity removing the instability in the reconstruction at the cost of producing artificially smooth images. A common choice for $\mathbf{R}$ is a discrete approximation to the Laplace operator, in which case the penalty term is a discrete approximation to

$$
G_{\text {Tikh }}(\sigma)=\int_{\Omega_{\text {in }}}\left|\nabla^{2} \sigma(x)\right|^{2} d V
$$

where $\Omega_{\text {in }}$ is the region of interest for the image.

The effect of Tikhonov regularization can be seen in Fig. 5, where the singular values cluster when the Tikhonov regularization matrix is applied to enforce a smoothness assumption to the conductivity distribution.

\section{B. Total Variation Regularization}

Although Tikhonov type regularization provides a good method to reconstruct smooth parameters both in terms of contrast and shape, it fails to reconstruct the sharp edges and absolute values for the high contrast case. TV regularization is a more suitable method for both sharp edges and high contrast. Using TV regularization to reconstruct the sharp edges has been discussed in [19] for EIT. The recovery of sharp edges in MIT using TV regularization is similar to the EIT problem. In other words, the TV functional is used to encourage blocky images as a regularized solution. The TV functional of a continuum $\sigma$

$$
G_{\mathrm{TV}}(\sigma)=\int_{\Omega_{\mathrm{in}}}|\nabla \sigma(x)| d V .
$$

Let the area of each facet $i$ between two tetrahedra be $q_{i}$ for $i=1,2, \ldots, I$. The $k$ th row of the matrix $\mathbf{S} \in \mathbb{R}^{I \times P}$ (here $I$ is number of facets and $P$ is number of tetrahedral elements) is chosen to be $\mathbf{S}_{k}=\left[\begin{array}{lll}0 \ldots 0 & 1 \ldots-1 & 0 \ldots 0\end{array}\right]$, where 1 and -1 occur in the columns corresponding to the tetrahedra with common facets $k$. Rows of $\mathbf{S}_{k}$ are then weighted with the surface area of $q_{k}$ of the facet $k$, and we define $\mathbf{R}_{\mathrm{TV}}=\mathbf{S}^{T} \mathbf{Q S}$, 

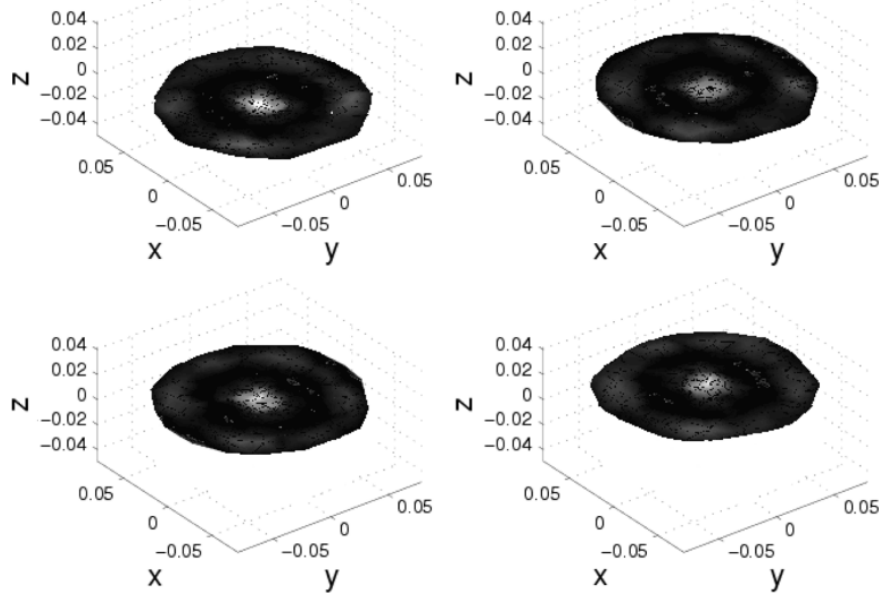

Fig. 6. Reconstruction of one metal bar presented at different cross sections. The heights are $z=-0.02$ for top left, $z=-0.01$ top right, $z=0.01$ bottom left, $z=0.02$ bottom right. Dimensions are in $\mathrm{m}$.
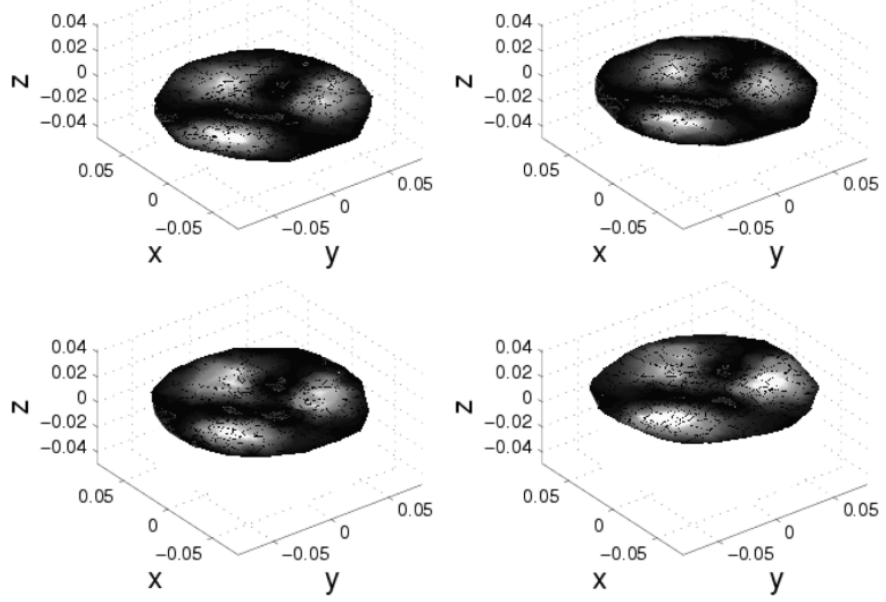

Fig. 7. Cross sections of reconstruction of three metal objects. Heights as Fig. 6.

where $\mathbf{Q}$ is a diagonal matrix with $Q_{k k}=q_{k}$. Using the 1-norm $\left\|\mathbf{R}_{\mathrm{TV}} \boldsymbol{\sigma}\right\|_{1}$ gives an approximation to the total variation function of the distribution $\sigma$ [25].

\section{RESULTS AND DISCUSSION}

\section{A. Experimental Tests}

Preliminary results using simulation data have been presented earlier. In [26], the simulation results for high contrast conductivity and in [27], the simulation results for low contrast conductivity (medical MIT) have been presented. In this paper, we are more concerned with validating the method with experimental data. Electrically conductive objects were tested using data from the MIT system. Test objects_rods and bars-used were $30 \mathrm{~cm}$ long, which we considered long compared to the $5 \mathrm{~cm}$ diameter of the detector element. These tests were arranged to represent typical liquid metal flow profiles such as central and multiple streams. Fig. 6 shows one object representing a single stream, where the image is well defined when a $9 \mathrm{~mm}$ diameter copper rod is placed at the center. Figs. 7 and 8 show the image results with multiple objects being tested representing multiple
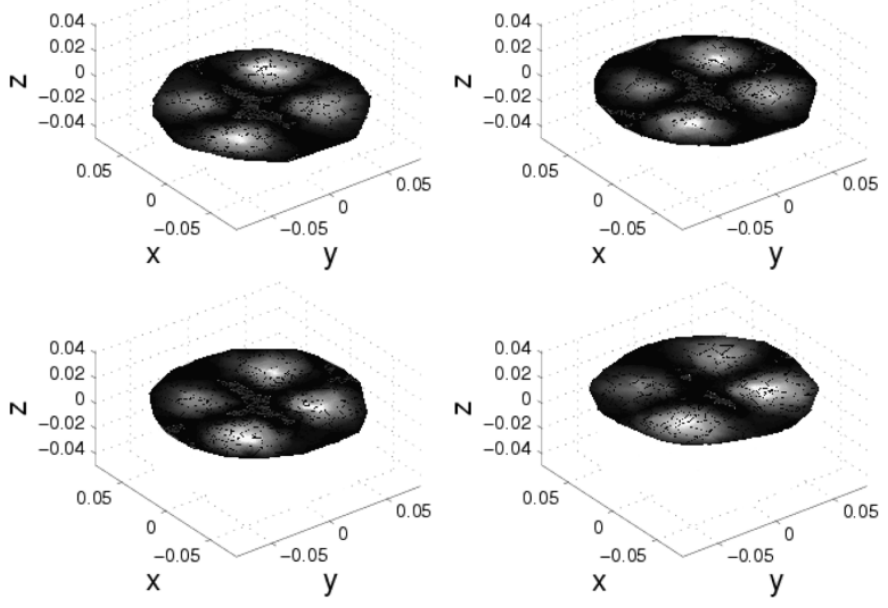

Fig. 8. Cross sections of reconstruction of four metal objects. Heights as Fig. 6.
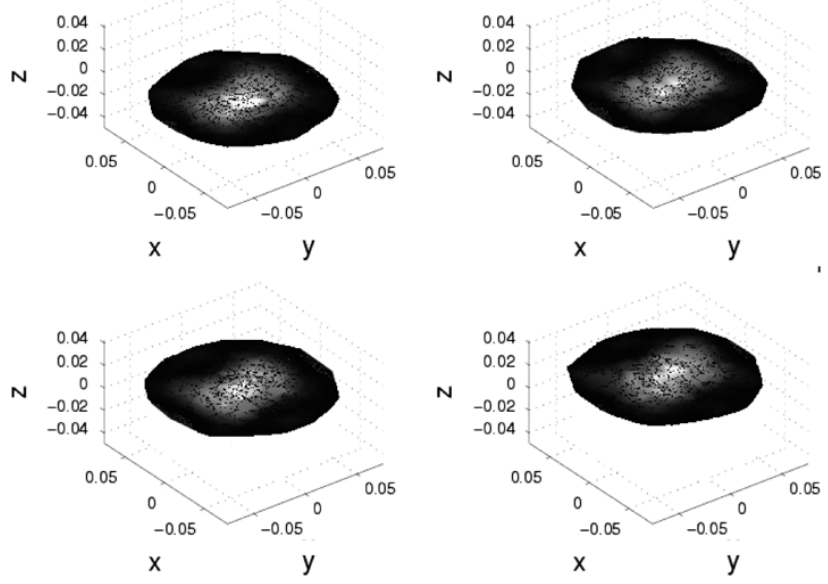

Fig. 9. Cross sections of reconstruction of a rectangular object in center. Heights as Fig. 6.

flow streams. In Fig. 7, three copper rods with $19 \mathrm{~mm}$ diameter were placed upright at the corners of an equilateral triangle. As shown in Fig. 8, three big copper rods (19 $\mathrm{mm}$ in diameter) and one small copper (12.5 $\mathrm{mm}$ in diameter) are clearly visible. An object with rectangular cross section was also tested, namely a rectangular box with a cross-sectional size of approximately $80 \mathrm{~mm} \times 50 \mathrm{~mm}$. Fig. 9 shows the reconstructed rectangular object using TV regularization. This showed better preservation of the sharp edges of the rectangular object than Tikhonov regularization. For smooth shape single objects or for objects far from each other, the TV and Tikhonov regularization produced similar images. Fig. 10 shows reconstruction of three copper bars close to each other (30 mm apart), using TV and Tikhonov regularization. In TV regularization, the separation between bars is improved compared to Tikhonov regularization. The number of iterations used for the inverse problem in these examples is less than six; further iterations were found not to improve the image quality. We have as yet found no fool-proof way to choose the regularization parameter for the nonlinear inverse problem. In this study we choose the regularization parameter using ad hoc methods, and this must always be treated with caution. The best one can hope for is to find a good parameter choice for a particular experimental setup and range of target objects. Further 

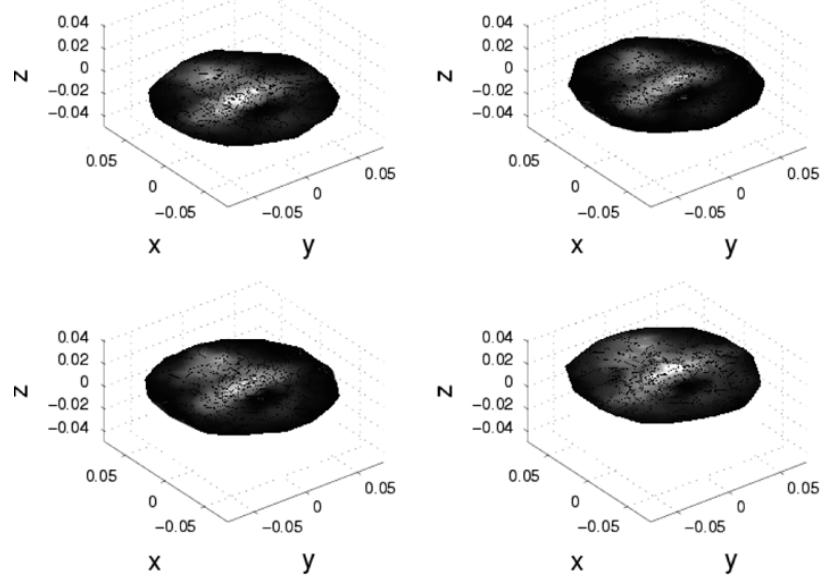

(a)
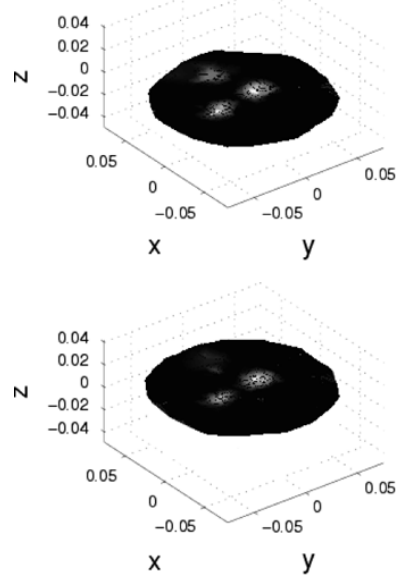

(b)
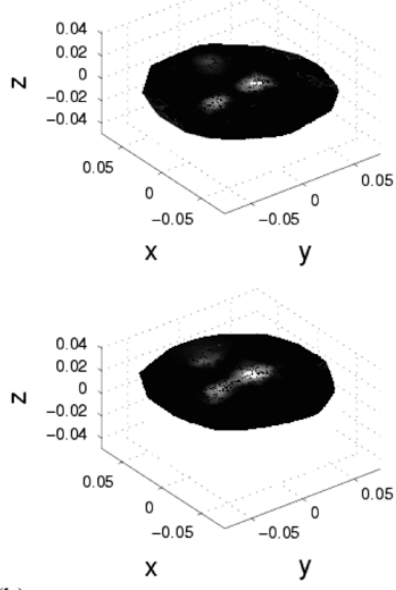

Fig. 10. Cross sections of reconstruction of three metallic objects close to each other using TV regularization and Tikhonov regularization. Heights as Fig. 6. (a) Tikhonov regularization. (b) Total variation regularization.

studies are required to find an automatic choice of regularization parameter in this nonlinear inverse problem. The regularization parameter was chosen $10^{-8}$ for Tikhonov regularization and $10^{-5}$ for TV regularization in all iterations.

In the MIT problem, the effect of high conductivity regions on measurements saturates as the conductivity increases. This is evident even in the modest conductivity contrasts in medical applications as discussed in [10]. For this reason reconstruction of the absolute values of the high contrast conductivities (quantitative image reconstruction) is a difficult, or perhaps an impossible, task both numerically and experimentally. Nonlinear reconstruction algorithms will typically result in a conductivity that fits the measurements to within the measurement or forward modeling error, but the conductivity contrast will be underestimated for large contrast objects. In our present work, we show the advantage of nonlinear image reconstruction in improving the quantitative estimation in that for high contrast objects the contrast in the reconstructed image is greater than for a linear step. However, a more important advantage we claim is an improvement in object localization. Taking the example of Fig. 8, the plot of Fig. 11 shows the reduction in the cost functional (8) for each iteration. Fig. 12 shows a cross section of the image at each iteration. In each iteration the separation between objects improved,

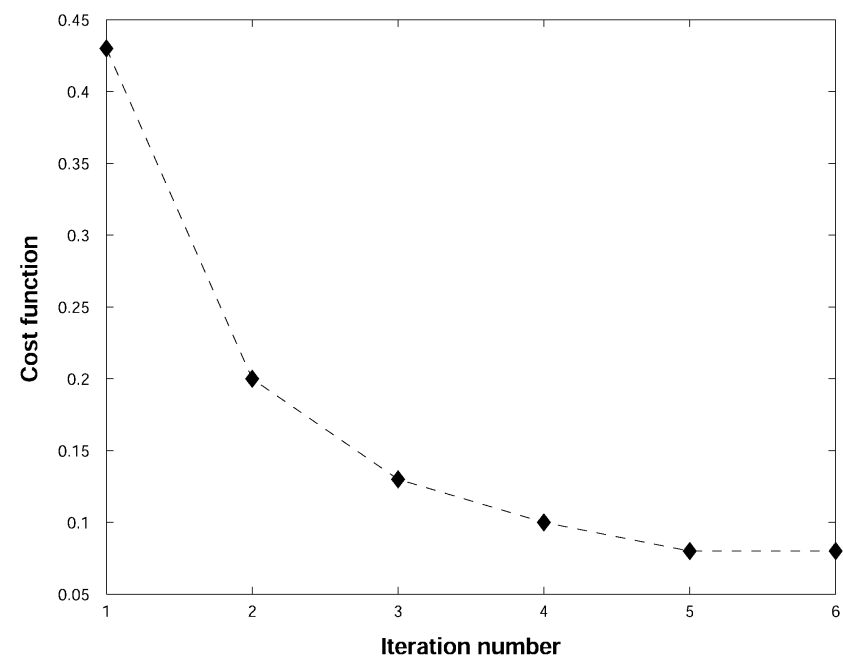

Fig. 11. Reduction in cost function with iteration steps.

and the contrast between the background and reconstructed conductivity of the rods increases up to six iterations at which saturation sets in and no further improvement is observed. Our explanation for this improvement in separation at each iteration is that in a nonlinear reconstruction algorithm, by recalculating the Jacobian matrix and forward model we account better for the interference of the secondary field between highly conductive objects. This is in contrast to the case of a single linear step that only considers the sensitivity at a fixed (homogeneous) background. We expect that the interference between highly conductive objects will increase with increasing the excitation frequency, and also the saturation will occur in lower conductivity values at higher frequency. The saturation and interference between near by objects is also a function of how close they are to the excitation coil—nearer to excitation coils, higher field strengths might be expected to produce a greater interference effect.

The edge FEM forward solver and the image reconstruction software have been written in Matlab. The overall number of tetrahedral elements in forward model was 86757 and the region of interest (Cylinder C1), which was used in inverse solution, included 3058 tetrahedral elements. The computer had a $1.7 \mathrm{GHz}$ Intel Pentium M processor and $512 \mathrm{MB}$ of RAM. The computational time for each nonlinear iteration of the inverse problem (consisting mainly of the forward solver, Jacobian calculation and inversion) was $26 \mathrm{~min}$. The major parts of the computational time includes solving two linear system of equations: one is for the forward problem involving a large but sparse matrix and the other one involves solution of a smaller but full matrix arising from the inverse problem.

\section{B. Flow Visualization in Continuous Casting}

Continuous casting is a process by which molten steel is formed into semi-finished billets, blooms, and slabs. Liquid steel from the basic oxygen steel-making or electric arc furnace process and subsequent secondary steel-making, is transferred from a ladle, via a refractory shroud, into the tundish. The tundish acts as a reservoir, both for liquid steel delivery and removal of oxide inclusions. A stopper rod or sliding gate is used to control the steel flow rate into the mould through a submerged entry nozzle (SEN). The SEN distributes the steel within the mould, shrouds the liquid steel from the surrounding environment, 


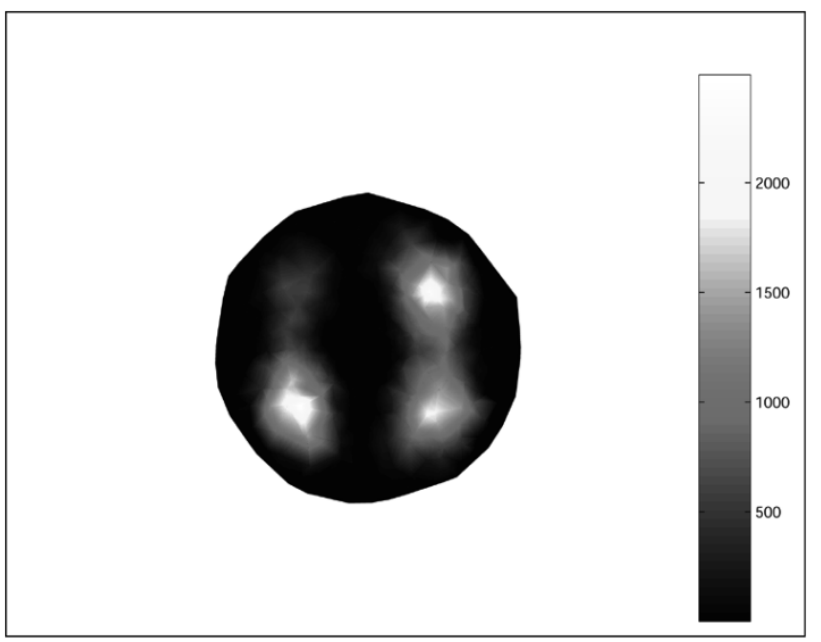

(a)

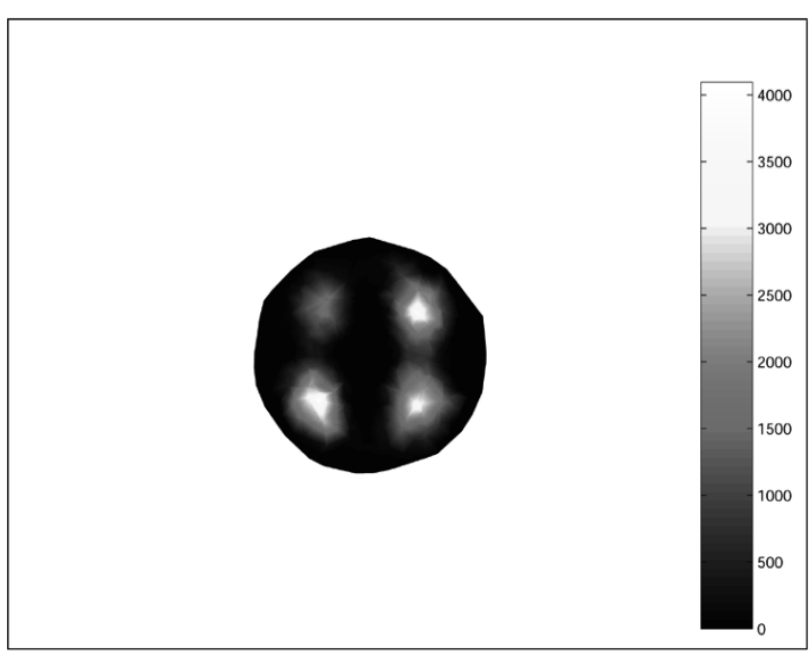

(b)

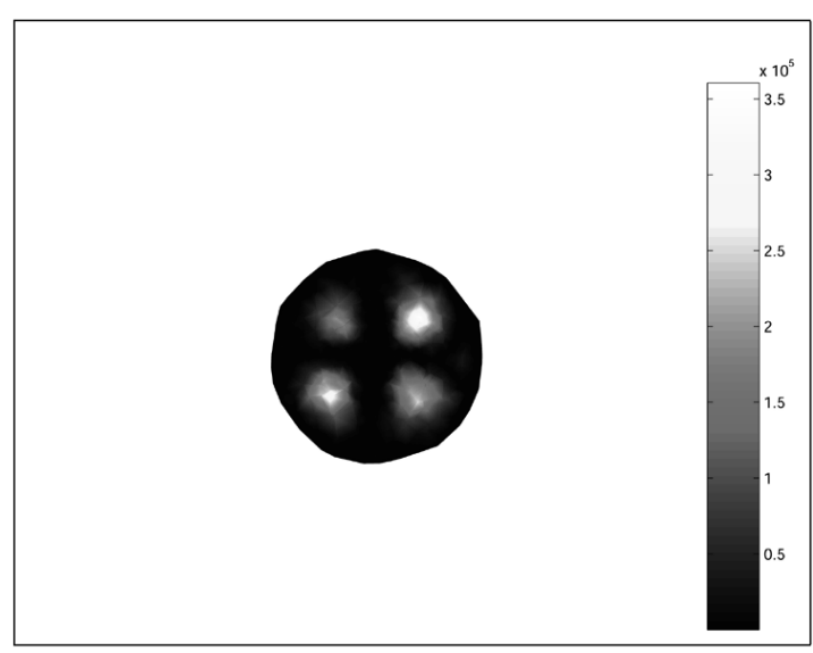

(c)

Fig. 12. Reconstruction of four metallic objects in iterations 1, 3, and 6. Note that they are displayed on different scales to show the improvement in object localization. The maximum conductivity values in the reconstructions are $2.25 \times$ $10^{3}$ for the first iteration, $4.18 \times 10^{3}$ third iteration and $3.65 \times 10^{5}$ sixth iteration. (a) Step 1. (b) Step 3. (c) Step 6.

and reduces air entrainment, thus preventing reoxidation and maintaining steel cleanliness. A photograph of real molten steel flow in continuous casting can be seen in Fig. 13.

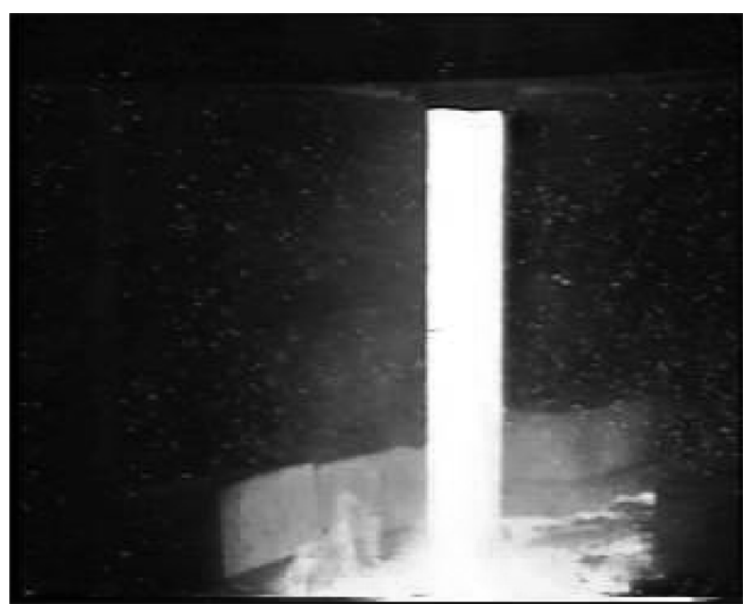

Fig. 13. Example of steel flow through a glass tube during a hot experiment.

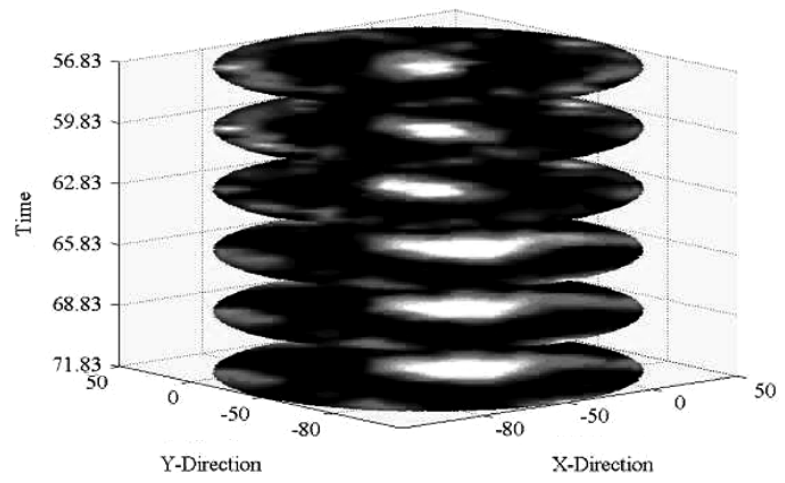

Fig. 14. Reconstruction of real steel flow, time is in seconds and $X$ and $Y$ here are in $\mathrm{mm}$, for each time step the image shows the $\mathrm{Z}=0$ level of 3 -D image.

The hot casting trials were undertaken at Corus RD\&T, Teesside Technology Centre. The sensor array was placed around a transparent quartz glass tube within a standard slab caster SEN and connected to the MIT instrument through long thermally shielded cables. Molten steel was supplied from a 4-ton nominal capacity electric arc furnace via a stoppered ladle to a tundish and then passed to a mock casting mould via the glass tube to enable the steel submerged pouring to be simulated. The outputs are arranged in a data matrix such that the rows represent the time sequence, i.e., image frame index, and the columns the coil-pair measurements. A selection of results is shown in Fig. 14. The images are shown in sequence from top to bottom indicating the pouring of molten steel at different time instants. The results were consistent with the real pouring arrangement in that pouring was initially positioned in the center and then positioned off-center. Consequentially, the hot trial results demonstrate the success of using electromagnetic sensing technique to monitor and visualize the real molten steel flow through a pouring nozzle.

\section{CONCLUSION}

In this paper, we described a general reconstruction method for 3-D magnetic induction tomography. Application of total variation regularization improves the separation between objects close to each other and recovery of sharp edges. The encouraging results from pilot plant trials suggest that the pro- 
posed MIT reconstruction method may be useful for monitoring and visualization of real molten steel flow through a pouring nozzle, although the stability of the hardware is an issue if the system is used for longer times in a hot environment. The proposed technique in this paper can be used in off-line image reconstruction in MIT, as the computational time can be very high. Further studies are required to improve the speed of the proposed technique for online monitoring. In particular improving the speed of the forward solver (for example by using algebraic multigrid solvers), use of parallel processing, and improvement in speed of the linear solvers for the inverse problem in each iteration step. Quantitative image reconstruction for high contrast conductivity applications, while desirable for some applications, is hard to achieve even with total variation regularization but reasonable qualitative results are possible. Further work is needed to both improve the accuracy of the experimental system and modeling in order to improve reconstruction accuracy.

\section{ACKNOWLEDGMENT}

This work was supported by the UK Engineering and Physical Sciences Research Council (EPRSC) under Grant GR/R64278/01 and Grant GR/R64285/01, and by Corus Group RD\&T, Teesside Technology Centre.

\section{REFERENCES}

[1] H. Griffiths, "Magnetic induction tomography," Meas. Sci. Technol., vol. 12 , no. 8, pp. 1126-1131, 2001.

[2] A. J. Peyton, Z. Z. Yu, S. Al-Zeibak, N. H. Saunders, and A. R. Borges, "Electromagnetic imaging using mutual inductance tomography: Potential for process applications," Part. Part. Syst. Charact., vol. 12, pp. 68-74, 1995.

[3] M. Pham, Y. Hua, and N. Gray, "Imaging the solidification of molten metal by eddy currents-Part I," Inverse Problems, vol. 16, pp. 483-494, Apr. 2000.

[4] H. Huang, T. Takagi, and H. Fukutomi, "Fast signal predictions of noised signals in eddy current testing," IEEE Trans. Magn., pt. 1, vol. 36, no. 4, pp. 1719-1723, Jul. 2000.

[5] Y. Li, L. Udpa, and S. S. Udpa, "Three-dimensional defect reconstruction from eddy-current NDE signals using a genetic local search algorithm," IEEE Trans. Magn., vol. 40, no. 2, pp. 410-417, Mar. 2004.

[6] S. J. Norton and J. R. Bowler, "Theory of eddy current inversion," $J$. Appl. Phys., vol. 73, no. 2, pp. 501-512, Jan. 15, 1993.

[7] G. Rubinacci, A. Tamburrino, and S. Ventre, "Regularization and numerical optimization of a fast eddy current imaging method," IEEE Trans. Magn., vol. 42, no. 4, pp. 1179-1182, Apr. 2006.

[8] R. Binns, A. R. A. Lyons, A. J. Peyton, and W. D. N. Pritchard, "Imaging molten steel flow profiles," Meas. Sci. Technol., vol. 12, pp. 1132-1138, 2001.

[9] A. V. Korjenevsky, V. A. Cherpenin, and S. Sapetsky, "Magnetic induction tomography: Experimental realization," Physiol. Meas., vol. 21, pp. 89-94, Feb. 2000.
[10] M. Soleimani and W. R. B. Lionheart, "Image reconstruction in three-dimensional magnetostatic permeability tomography," IEEE Trans. Magn., vol. 41, no. 4, pp. 1274-1279, Apr. 2005.

[11] O. Biro, "Edge element formulations of eddy current problems," Comput. Meth. Appl. Mech. Eng., vol. 169, pp. 391-405, 1999.

[12] R. Merwa, K. Hollauhs, B. Brandstatter, and H. Scharfetter, "Numerical solution of the general 3D eddy current problem for magnetic induction tomography (spectroscopy)," Physiol. Meas., vol. 24, no. 2, pp. 545-554, 2003.

[13] M. Soleimani, W. R. B. Lionheart, C. H. Riedel, and O. Dossel, "Forward problem in 3D magnetic induction tomography (MIT)," in Proc. 3rd World Congr. Industrial Process Tomography, The Rockies, AB, Canada, 2003, pp. 275-280.

[14] W. R. B. Lionheart, M. Soleimani, and A. J. Peyton, "Sensitivity analysis in 3D magnetic induction tomography (MIT)," in Proc. 3rd World Congr. Industrial Process Tomography, Banff, AB, Canada, 2003, pp. 239-244.

[15] O. Dorn, H. Bertete-Aguirre, J. G. Berryman, and G. C. Papanicolaou, "A nonlinear inversion method for 3D electromagnetic imaging using adjoint fields," Inverse Problems, vol. 15, pp. 1523-1558, 1999.

[16] D. N. Dyck, D. A. Lowther, and E. M. Freeman, "A method of computing the sensitivity of the electromagnetic quantities to changes in the material and sources," IEEE Trans. Magn., vol. 30, no. 5, pp. 3415-3418, Sep. 1994.

[17] C. R. Vogel, "Computational methods for inverse problems," in Frontiers Appl. Math., 2002, SIAM.

[18] T. F. Chan and X. C. Tai, "Level set and total variation regularization for elliptic inverse problems with discontinuous coefficients," J. Comput. Phys., vol. 193, no. 1, pp. 40-66, 2004.

[19] A. Borsic, W. R. B. Lionheart, and C. N. McLeod, "Generation of anisotropic-smoothness regularization filters for EIT," IEEE Trans. Med. Imag., vol. 21, pp. 579-587, Jun. 2002.

[20] X. Ma, S. R. Higson, A. Lyons, and A. J. Peyton, "Development of a fast electromagnetic induction tomography system for metal process applications," in Proc. 4th World Congr. Industrial Process Tomography, Aizu, Japan, 2005, pp. 196-201.

[21] B. A. Auld, Eddy-Current Characterization of Materials and Structures, G. Birnbaum and G. Free, Eds., 1981, pp. 332-347. ASTM STP 722.

[22] H. Scharfetter, P. Riu, M. Populo, and J. Rosell, "Sensitivity maps for low-contrast-perturbations within conducting background in magnetic induction tomography (MIT)," Physiol. Meas., vol. 23, pp. 195-202, 2002.

[23] S. Watson, R. J. Williams, H. Griffiths, W. Gough, and A. Morris, "Magnetic induction tomography: Phase versus vector-voltmeter measurement techniques," Physiol. Meas., vol. 24, pp. 555-564, 2003.

[24] J. Qi-Nian, "On the iteratively regularised Gauss-Newton method for solving nonlinear ill-posed problems," Math. Comput., vol. 69, no. 232, pp. 1603-1623, 2000.

[25] D. C. Dobson and F. Santosa, "An image enhancement technique for electrical impedance tomography," Inverse Problems, vol. 10, pp. 317-334, 1994.

[26] M. Soleimani, W. R. B. Lionheart, A. J. Peyton, and X. Ma, "Image reconstruction in 3D magnetic induction tomography using a FEM forward model," in Proc. 3rd World Congr. Industrial Process Tomography, The Rockies, AB, Canada, Sep. 2003, pp. 252-255.

[27] M. Soleimani and W. R. B. Lionheart, "Image reconstruction in magnetic induction tomography using a regularized Gauss Newton method," in Proc. XII. Int. Conf. Electrical Bio-Impedance V. Electrical Impedance Tomography, Gdansk, Poland, 2004, pp. 551-554.

Manuscript received June 10, 2005; revised February 1, 2006. Corresponding author: W. R. B. Lionheart (e-mail: Bill.Lionheart@manchester.ac.uk). 